This article was downloaded by: [National Chiao Tung University 國立交通大學]

On: 25 April 2014, At: 04:35

Publisher: Taylor \& Francis

Informa Ltd Registered in England and Wales Registered Number: 1072954 Registered office: Mortimer House, 37-41 Mortimer Street, London W1T 3J H, UK

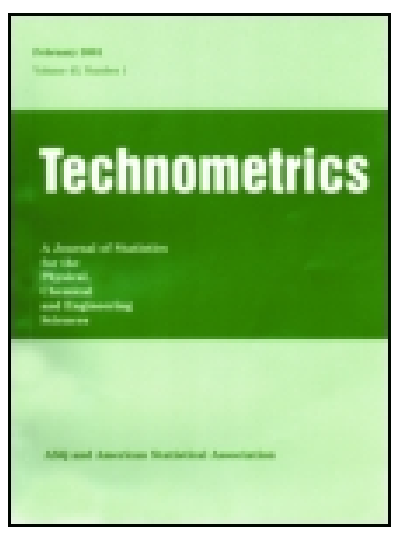

\title{
Technometrics
}

Publication details, including instructions for authors and subscription information: http:// www. tandfonline.com/loi/ utch20

\section{Tolerance Intervals With Improved Coverage Probabilities for Binomial and Poisson Variables}

\author{
Hsiuying Wang ${ }^{a} \&$ Fugee Tsung $^{b}$ \\ ${ }^{a}$ Institute of Statistics, National Chiao Tung University, Hsinchu, Taiwan \\ ${ }^{\mathrm{b}}$ Department of Industrial Engineering and Engineering Management, Hong Kong \\ University of Science and Technology, Kowloon, Hong Kong, China \\ Published online: $01 \mathrm{~J}$ an 2012.
}

To cite this article: Hsiuying Wang \& Fugee Tsung (2009) Tolerance Intervals With Improved Coverage Probabilities for Binomial and Poisson Variables, Technometrics, 51:1, 25-33, DOI: 10.1198/TECH.2009.0003

To link to this article: http:// dx. doi. org/ 10.1198/TECH.2009.0003

\section{PLEASE SCROLL DOWN FOR ARTICLE}

Taylor \& Francis makes every effort to ensure the accuracy of all the information (the "Content") contained in the publications on our platform. However, Taylor \& Francis, our agents, and our licensors make no representations or warranties whatsoever as to the accuracy, completeness, or suitability for any purpose of the Content. Any opinions and views expressed in this publication are the opinions and views of the authors, and are not the views of or endorsed by Taylor \& Francis. The accuracy of the Content should not be relied upon and should be independently verified with primary sources of information. Taylor and Francis shall not be liable for any losses, actions, claims, proceedings, demands, costs, expenses, damages, and other liabilities whatsoever or howsoever caused arising directly or indirectly in connection with, in relation to or arising out of the use of the Content.

This article may be used for research, teaching, and private study purposes. Any substantial or systematic reproduction, redistribution, reselling, loan, sub-licensing, systematic supply, or distribution in any form to anyone is expressly forbidden. Terms \& Conditions of access and use can be found at http:// www.tandfonline.com/page/terms-and-conditions 


\title{
Tolerance Intervals With Improved Coverage Probabilities for Binomial and Poisson Variables
}

\author{
Hsiuying WANG \\ Institute of Statistics \\ National Chiao Tung University \\ Hsinchu, Taiwan
}

\author{
Fugee Tsung \\ Department of Industrial Engineering and \\ Engineering Management \\ Hong Kong University of Science and Technology \\ Kowloon, Hong Kong, China
}

\begin{abstract}
The construction of tolerance intervals (TIs) for discrete variables, such as binomial and Poisson variables, has been critical in industrial applications in various sectors, including manufacturing and pharmaceuticals. Inaccurate estimation of coverage probabilities leads to improper construction of tolerance intervals and may lead to serious financial losses for the manufacturers. This article proposes procedures to compute the exact minimum and average coverage probabilities of the tolerance intervals for Poisson and binomial variables. These procedures are illustrated with examples and real data applications. Based on these procedures, improved tolerance intervals are proposed that can ensure that the true minimum or average coverage probabilities are very close to the nominal levels.
\end{abstract}

KEY WORDS: Binomial distribution; Poisson distribution; Quality control; Tolerance interval.

\section{INTRODUCTION}

The construction of tolerance intervals (TIs) to measure discrete quality characteristics has been one of the major tasks in developing quality control systems used in the manufacturing and pharmaceutical sectors. Hahn and Chandra (1981) investigated a general problem on controlling the number of unscheduled shutdowns of a complex system. The manufacturer needs to construct an upper tolerance bound on the maximum number of unscheduled shutdowns that can be expected, with $95 \%$ confidence, to occur in $90 \%$ of the systems in 1 year of operation. If the estimation is inaccurate and the true coverage probability of the constructed TI is underestimated, this might cause the company to ignore necessary improvement measures, leading to serious financial losses. Examples that require an accurate construction of TI for discrete data are found in many industrial practices. In this article we investigate in more detail a case on constructing TI to control the occurrence of surface defects in a steel manufacturing process that follows a Poisson distribution and another case on constructing TI to control the number of defective wafers in a semiconductor manufacturing process that follows a binomial distribution. Other applications have been presented by Hahn and Meeker (1991) and Hahn and Chandra (1981).

Although the construction of TIs is as important for discrete distributions as it is for continuous distributions, there has been much less work on this type of problem compared with the developments on continuous distributions (see, e.g., Wald and Wolfowitz 1946; Odeh and Owen 1980; Wang and Iyer 1994; Van Der Merwe, Pretorius, and Meyer 2006; Wolfinger 1998; Hamada et al. 2004; Liao, Lin, and Iyer 2005; Krishnamoorthy and Mathew 2004; Fernholz and Gillespie 2001).

Nevertheless, Zacks (1970) proposed a criterion for selecting tolerance limits for monotonic likelihood ratio families of discrete distributions. The tolerance limits chosen according to this criterion are called the uniformly most accurate tolerance limits. After that, the most widely used TIs for Poisson and binomial variables were constructed by Hahn and Chandra (1981). A survey of these intervals was presented by Hahn and Meeker
(1991). The TIs were constructed by a two-step process: (1) Find a confidence interval of the unknown parameter, and (2) use the bounds of the confidence interval to obtain the tolerance bounds.

For a binomial or Poisson distribution with an unknown parameter, $\theta$, the level $(1-\alpha)$ of the confidence interval for $\theta$ are usually determined by a large-sample approximate method. TIs are then constructed based on these confidence intervals. When the sample size is large, the true coverage probability of the TIs at a fixed point in the parameter space may be close to the nominal level. But when the sample size is not large, the true coverage probability of the TI may be far from the nominal coefficient. Indeed, the TIs constructed by conventional approaches frequently overcover or undercover, as we demonstrate later. If the TIs do not overcover, then only a small proportion of units of the product meet the requirement, which can cause not only profit loss, but also damage to the manufacturer's good name. On the other hand, TIs often can undercover as well; that is, the TIs or bounds are too conservative. As a consequence, a customer may not accept a product because the upper tolerance bound is too large or the lower tolerance bound is too small, even though the product quality is indeed acceptable. Therefore, establishing an approach to construct a TI with an accurate coverage probability is essential in practice.

Although this is an important problem, it is technically challenging to calculate exact coverage probabilities and derive precise TIs for discrete distributions. A similar problem has arisen for confidence intervals because the minimum coverage probability of a confidence interval for discrete distributions also is usually unknown. Wang (2007) proposed an approach to calculating the minimum coverage probability for binomial confidence intervals that can exactly derive the point in the parameter space in which the minimum coverage probability occurs, as well as the minimum coverage probability.

(c) 2009 American Statistical Association and the American Society for Quality TECHNOMETRICS, FEBRUARY 2009, VOL. 51, NO. 1 DOI 10.1198/TECH.2009.0003 
Because the minimum coverage probability is an important index for evaluating the performance of TIs, it has been estimated through a statistical simulation approach. The simulation method computes the coverage probabilities at some randomly chosen points in the parameter space and constructs an estimator based on the minimum value of these coverage probabilities. But this simulation method obtains only a roughly approximated value, not an exact value. In addition to the disadvantage of the rough estimate of the minimum coverage probability, the simulation method also is more time-consuming. It requires calculation of more coverage probabilities than our proposed method. To date, no methodology for computing the exact minimum coverage probability for TIs of discrete distributions has been reported in the literature; to the best of our knowledge, our proposed approach is the first attempt to demonstrate this methodology.

We propose novel procedures for calculating the minimum and average coverage probabilities of TIs for binomial and Poisson distributions. With these procedures, we are able to obtain improved TIs with their true minimum or average coverage probabilities very close to the nominal levels. The article is organized as follows. The most widely used TIs for binomial and Poisson distributions are introduced in Section 2. The methods for computing the minimum and average coverage probabilities of TIs are given in Sections 3 and 4. These methods are illustrated by numerical examples in Section 5. Improved TIs with required minimum or average coverage probabilities are proposed in Section 6. In Section 7 two industrial applications from the manufacturing industry are used to demonstrate the applicability of the proposed procedures and the improved tolerance intervals. The article concludes in Section 8 with a summary of our contributions and some concluding remarks.

\section{TOLERANCE INTERVALS}

The TIs for discrete variables are widely used in industrial applications in which the quality characteristics (e.g., the number of defective parts) require specific bounds (i.e., the TI) for the purpose of control and surveillance. Let $F$ denote the cumulative distribution for a random variable, $X$. An interval, $(L(X), U(X))$, is said to be a $\beta$-content, $1-\alpha$ confidence TI, denoted as a $(\beta, 1-\alpha)$ TI, for $F$ if

$$
\operatorname{Pr}_{\theta}\{[F(U(X))-F(L(X))] \geq \beta\}=1-\alpha .
$$

The TIs give us $L$ and $U$ such that we can claim, with a specified degree of confidence, $1-\alpha$, that a specified proportion, $\beta$, or more of the manufactured items lie between $L$ and $U$.

For a continuous distribution, there may exist a TI, $(L(X)$, $U(X))$, satisfying (1) for all $\theta$. But for discrete distributions, the value of the left side of (1) depends on the parameter $\theta$. Therefore, for a fixed $\beta$, the left side of (1) is not a constant. In this situation, it is reasonable to modify the definition of (1) for a discrete distribution as follows:

$$
\operatorname{Pr}_{\theta}\{[F(U(X))-F(L(X))] \geq \beta\} \geq 1-\alpha,
$$

and there exists a $\theta$ such that the equality holds.

According to Hahn and Meeker (1991) and Hahn and Chandra (1981), the construction of a $(\beta, 1-\alpha)$ TI for a binomial or Poisson distribution with an unknown parameter, $\theta$, for the observed value, $x$, of $X$ is based on the following two steps:
1. Construct a two-sided $(1-\alpha)$ confidence interval $(l, u)$ for $\theta$, where $l$ and $u$ depend on $x$.

2. Find a minimum number, $U(x)$, and a maximum number, $L(x)$, such that

$$
\begin{aligned}
& \operatorname{Pr}_{u}(X \leq U(x)) \geq(1+\beta) / 2 \quad \text { and } \\
& \operatorname{Pr}_{l}(X \geq L(x)) \geq(1+\beta) / 2 .
\end{aligned}
$$

The one-sided tolerance bounds can be obtained in a similar way. An upper $(\beta, 1-\alpha)$ tolerance interval is constructed by finding an upper $(1-\alpha)$ confidence bound of $\theta$, say $u$, and then deriving the minimum number, $U(X)$, such that $p_{u}(X \leq$ $U(X)) \geq \beta$. The interval $(0, U(X))$ is used as an upper $(\beta$, $1-\alpha)$ tolerance bound. A lower $(\beta, 1-\alpha)$ TI is constructed by finding a lower $(1-\alpha)$ confidence bound of $\theta$, say $l$, and then deriving the maximum number, $L(X)$, such that $p_{l}(X \geq$ $L(X)) \geq \beta$. The intervals $(L(X), n)$ and $(L(X), \infty)$ are used as lower $(\beta, 1-\alpha)$ tolerance bounds for the binomial and Poisson distributions.

For the binomial distribution, the suggested $(1-\alpha)$ confidence intervals for $\theta$ in (I) introduced by Hahn and Meeker (1991) are

$$
(l, u)=\hat{\theta} \pm z_{(1-\alpha / 2)}\left(\frac{\hat{\theta}(1-\hat{\theta})}{n}\right)^{1 / 2}
$$

and

$$
\begin{gathered}
(l, u)=\left(\left(1+\frac{(n-x+1) F_{(1-\alpha / 2 ; 2 n-2 x+2,2 x)}}{x}\right)^{-1},\right. \\
\left.\left(1+\frac{n-x}{(x+1) F_{(1-\alpha / 2 ; 2 x+2,2 n-2 x)}}\right)^{-1}\right),
\end{gathered}
$$

where $z_{a}$ and $F_{\left(a ; r_{1}, r_{2}\right)}$ are the $(100 a)$ th percentile of the standard normal distribution and the $(100 a)$ th percentile of the $F$ distribution with $r_{1}$ and $r_{2}$ degrees of freedom.

For the Poisson distribution, the suggested $(1-\alpha)$ confidence intervals in Step 1, introduced by Hahn and Meeker (1991), are

$$
(l, u)=\hat{\theta} \pm z_{(1-\alpha / 2)}\left(\frac{\hat{\theta}}{n}\right)^{1 / 2}
$$

and

$$
(l, u)=\left(0.5 \chi_{(\alpha / 2 ; 2 x)}^{2} / n, 0.5 \chi_{(1-\alpha / 2 ; 2 x+2)}^{2} / n\right),
$$

where $\chi_{\left(a ; r_{1}\right)}^{2}$ is the $(100 a)$ th percentile of the chi-squared distribution with $r_{1}$ degrees of freedom. Note that (6) is defined as $\left(0,0.5 \chi_{(1-\alpha / 2 ; 2 x+2)}^{2} / n\right)$ if $x=0$.

Intervals (3) and (4) are the Wald and exact confidence intervals for the binomial proportion, and intervals (5) and (6) are the Wald and exact confidence intervals for the Poisson mean. The Wald intervals are derived by the large-sample approximation theory. They do not have satisfactory performance even with a large sample size. It is well known that the score confidence interval is better than the Wald interval (see Agresti and Coull 1998; Brown, Cai, and DasGupta 2001). But even if we use the score interval to construct the TI, its coverage probability still cannot be very close to the nominal level. Therefore, one goal of this article is to provide methods for calculating their exact minimum and the average coverage probabilities. 


\section{MINIMUM COVERAGE PROBABILITY}

In a discrete distribution, the coverage probability of a TI is a variable function of the parameter, $\theta$. For a claimed $\beta$-content, the $(1-\alpha)$-level TI, the true coverage probability depends on the true parameter. Because the true parameter value is unknown, we cannot know the true coverage probability; however, we do know that it is greater than the minimum coverage probability. If the minimum coverage probability can be derived, then the true coverage probability is not less than this value regardless of the value of the true parameter. Thus it can provide at least a conservative estimate of the coverage probability.

In this section we develop a procedure to calculate the exact minimum coverage probabilities of the TIs for binomial and Poisson distributions. Let $f_{\theta}(x)$ be the probability function of the binomial or Poisson variable. The minimum coverage probabilities are calculated as follows.

Procedure 1 (Computing the minimum coverage probability). For a $(\beta, 1-\alpha) \mathrm{TI},(L(X), U(X))$, satisfying $L(X)<L(Y)$ and $U(X)<U(Y)$ for $X<Y$, the minimum coverage probability can be obtained through the following steps:

Step 1. For each observation, $x, x=0, \ldots, n$, make $g_{x}(\theta)=$ $\sum_{i=L(x)}^{U(x)} f_{\theta}(i)$.

Step 2. Calculate the solutions of $g_{x}(\theta)=\beta$ for each $x$. There may be zero, one, or two solutions. The number of solutions depends on $\beta$ and $x$. Assume that there are totally $k$ solutions for all $x=0, \ldots, n$.

Step 3. Rank all $k$ solutions in Step 2. Let $v_{i}$ be the $i$ th smallest solution in Step 2.

Step 4. Compute the probability, $\operatorname{Pr}_{v_{i}}(F(U(Y))-F(L(Y))>$ $\beta$ ), for each $i$, which is the sum of the probabilities of $y$ such that $F(U(Y))-F(L(Y))>\beta$. The smallest value among these probabilities, $\operatorname{Pr}_{v_{i}}(F(U(Y))-F(L(Y))>\beta), v_{i}=1, \ldots, k$, is the minimum coverage probability of the TI.

Note that Step 1 must be modified for the Poisson distribution, where the sample space is infinite. In practical applications, we can use estimates of the expected value to select a value of $n(<\infty)$ such that the probability that the Poisson variable exceeds this $n$ is negligible. This $n$ is an effective upper bound on the distribution. We then apply Procedure 1 using this value of $n$ in Step 1.

The proof of this procedure is given in Theorem 1. Before proving Theorem 1, we need the following notations and lemmas.

Proposition 1. For the probability function, $f_{\theta}(x)$, of the binomial distribution or Poisson distribution, (a) $\sum_{i=L(x)}^{U(x)} f_{\theta}(i)$ is a decreasing function or an increasing function of $\theta$ when $L(x)=0$ or $U(x)=n$ and (b) $\sum_{i=L(x)}^{U(x)} f_{\theta}(i)$ is a unimodal function when $0<L(x)<U(x)<n$.

The proof of Proposition 1 for the binomial distribution is given in lemma 1 of Wang (2007). The Poisson distribution case can be proved by a similar argument. By Proposition 1, there are at most two solutions of $\sum_{i=L(x)}^{U(x)} f_{\theta}(i)=\beta$. The number of solutions depends on $\beta$ and on whether the function $\sum_{i=L(x)}^{U(x)} f_{\theta}(i)$ is an increasing, a decreasing, or a unimodal function. When
$\sum_{i=L(k)}^{U(k)} f_{\theta}(i)$ is a unimodal function, there are at most two solutions of $\theta$ for $\sum_{i=L(k)}^{U(k)} f_{\theta}(i)$ set to $\beta$, say $p_{k}$, and $q_{k}$. When $\sum_{i=L(k)}^{U(k)} f_{\theta}(i)$ is a decreasing function, there is at most one solution of $\theta$ for $\sum_{i=L(k)}^{U(k)} f_{\theta}(i)$ set to $\beta$, say $q_{k}$, and $p_{k}$ is defined as 0 . When $\sum_{i=L(k)}^{U(k)} f_{\theta}(i)$ is an increasing function, there is at most only one solution for $\theta$ for $\sum_{i=L(k)}^{U(k)} f_{\theta}(i)$ set to $\beta$, say $p_{k}$, and $q_{k}$ is defined as 1 . Note that if $\beta$ is very close to 1 , then there may be no solutions for $\sum_{i=L(k)}^{U(k)} f_{\theta}(i)$ set to $\beta$.

Lemma 1. Assume that $L(X)<L(Y)$ and $U(X)<U(Y)$ if $X<Y$. For any $k$ and $l, k<l$, the equation $\sum_{i=L(k)}^{U(k)} f_{\theta}(i)=$ $\sum_{i=L(l)}^{U(l)} f_{\theta}(i)$ has only one solution.

Lemma 2. Assume that $L(X)<L(Y)$ and $U(X)<U(Y)$ if $X<Y$. For $\beta$, if $p_{x}, p_{z}, p_{y}, q_{x}, q_{z}$, and $q_{y}$ exist, then $p_{x} \leq p_{z} \leq$ $p_{y}$, and $q_{x} \leq q_{z} \leq q_{y}$ if $x<z<y$.

Theorem 1. For a TI, $(L(X), U(X))$, of a discrete distribution satisfying

$$
L(X)<L(Y), \quad U(X)<U(Y) \quad \text { if } X<Y,
$$

the exact coverage probability is the minimum value of the $k$ probabilities

$$
\left\{\operatorname{Pr}_{\theta}(F(U(Y))-F(L(Y))>\beta): \theta \in W=\left\{v_{1}, \ldots, v_{k}\right\}\right\},
$$

where $k$ is the number of $v_{i}$, which is one of the existing $p_{i}$ and $q_{i}, i=0, \ldots, n$ and $v_{1}<\cdots<v_{k}$.

The proofs of Lemmas 1 and 2 and Theorem 1 are given in the Appendix. The procedure for computing the minimum coverage probability is based on Theorem 1 .

Remark 1. In Theorem 1 , if $\beta$ is very close to 1 such that there do not exist any solutions, $p_{i}$ and $q_{i}$, then the coverage probability is zero.

Remark 2. If the parameter space is known to be a restricted parameter space, then Procedure 1 still holds by replacing the set of solutions in Step 2 by the subset of the solutions belonging to the restricted parameter space. The minimum coverage probability is the minimum value of the coverage probabilities at $\theta$ in the subset.

\section{EXACT AVERAGE COVERAGE PROBABILITY}

Instead of reporting a minimum coverage probability, some researchers are interested in exploring average coverage probabilities. An average coverage probability can reflect the overall performance of a TI under a given prior on the parameter space. Compared with the minimum coverage probability, which is the behavior at a point or at several points in the parameter space, the average coverage probability can provide a more objective evaluation of a TI.

Let $\eta(\theta)$ be a prior on the parameter space, $\Omega$. The average coverage probability under $\eta(\theta)$ is defined as

$$
\int_{\Omega} \operatorname{Pr}_{\theta}(F(U(y))-F(L(y))>\beta) \eta(\theta) d \theta .
$$

This value takes the average of the coverage probability with respect to a prior, $\eta(\theta)$. For the binomial distribution, the prior is 
usually chosen to be the uniform prior, $\eta(\theta)=1$ for $\theta \in(0,1)$. For the Poisson distribution, although the natural parameter space is unbounded, it is reasonable to assume that the parameter space is bounded in real applications. The prior can be chosen to be the uniform prior or another prior in the bounded parameter space. In this article we use the uniform prior for the examples.

For some TIs, the minimum coverage probability may be far below the nominal level, but the average coverage probability could be much higher than the minimum coverage probability. As in the study of the minimum coverage probability, the literature reports no procedures for calculating the exact average coverage probability. The average coverage probability is usually approximated by the simulation approach, which can provide only a rough estimation. The procedure for computing the average coverage probability proposed in this article certainly provides an accurate way to solve this problem.

We give some notation and definitions before describing the procedure for computing the average coverage probability. Note that $v_{i}$ is defined as a $p_{x}$ or $q_{x}$. If $v_{i}$ is a $p_{x}$ for some $x$, then let $r_{i}$ be the smallest $y$ such that $q_{y}>p_{x}$. If $v_{i}$ is a $q_{x}$ for some $x$, then let $s_{i}$ be the largest $y$ such that $p_{y} \leq q_{x}$.

Let

$$
h_{i}(\theta)= \begin{cases}\sum_{j=r_{i}}^{x} f_{\theta}(j) & \text { if } v_{i} \text { is a } p_{x} \\ \sum_{j=x+1}^{s_{i}} f_{\theta}(j) & \text { if } v_{i} \text { is a } q_{x} .\end{cases}
$$

Note that the parameter space can be separated into $(k+1)$ intervals by the points $v_{1}, \ldots, v_{k}$. For the Poisson distribution, $k$ may be infinity.

Theorem 2. For $\theta$ in the interval $\left(v_{i}, v_{i+1}\right), i=0, \ldots, k$ in the parameter space, the probability function,

$$
\operatorname{Pr}_{\theta}(F(U(Y))-F(L(Y))>\beta),
$$

is $h_{i}(\theta)$, where $v_{0}$ and $v_{k+1}$ are the lower endpoint and the upper endpoint of the parameter space.

The proof of Theorem 2 is given in the Appendix, which leads to the following procedure.

Procedure 2 (Computing the exact average coverage probability). For a $(\beta, 1-\alpha)$ TI satisfying $L(X)<L(Y)$ and $U(X)<$ $U(Y)$ for $X<Y$, the average coverage probability under the prior $\eta(\theta)$ can be derived by the following four steps:

Steps 1-3. Follow the same steps as in Procedure 1.

Step 4. Compute the summation

$$
S=\sum_{i=0}^{k} \int_{v_{i}}^{v_{i+1}} h_{i}(\theta) \eta(\theta) d \theta,
$$

which is the exact average coverage probability of the $(\beta, 1-\alpha)$ TI.

Remark 3. If the parameter space is known to be a restricted parameter space, then Procedure 2 still holds by replacing the solutions in Step 2 with the subset of the solutions belonging to the restricted parameter space. The average coverage probability is

$$
\begin{aligned}
S_{1}=\int_{a}^{v_{o_{1}}} h_{o_{1}}(\theta) \eta(\theta) d \theta & \\
& +\sum_{i=o_{1}}^{o_{2}} \int_{v_{i}}^{v_{i+1}} h_{i}(\theta) \eta(\theta) d \theta+\int_{v_{o_{2}}}^{b} h_{o_{2}+1}(\theta) \eta(\theta) d \theta
\end{aligned}
$$

where $a$ and $b$ are the lower and upper endpoints of the restricted parameter space, and $v_{o_{1}}, v_{o_{1}+1}, \ldots, v_{o_{2}}$ are the solutions in Step 2 belonging to the interior of the restricted parameter space.

\section{NUMERICAL RESULTS}

The procedures in Sections 3 and 4 help us derive the minimum coverage probability and the average coverage probability of the TIs for a binomial or Poisson distribution. The procedures for calculating the minimum coverage probability and the average coverage probability are illustrated by an example of $n=10$ for the binomial distribution.

Example 5.1. For $n=10$, the $(0.9,0.95)$ two-sided tolerance intervals based on the usual 0.95 confidence interval (3) are $(0,0),(0,5),(0,7),(0,8),(0,9),(0,10),(1,10),(2,10)$, $(3,10),(5,10)$, and $(10,10)$ corresponding to $x=0,1, \ldots, 10$. The 11 functions, $g_{x}(\theta)$, corresponding to $x=0, \ldots, 10$ are $\sum_{i=0}^{0} l_{\theta}(i), \sum_{i=0}^{5} l_{\theta}(i), \ldots, \sum_{i=10}^{10} l_{\theta}(i)$, where $l_{\theta}(i)=\left(\begin{array}{c}10 \\ i\end{array}\right) \times$ $p^{i}(1-p)^{n-i}$. The solutions for $g_{x}(\theta)$ set to $\beta=0.9$ are $0.0105,0.3542,0.5504,0.6632$, and 0.7943 , corresponding to $x=0, \ldots, 4$. There are no solutions for $g_{x}(\theta)$ set to 0.9 for $x=5$, because its corresponding $g_{x}(\theta)$ is 1 . The solutions of $g_{x}(\theta)$ set to $\beta=0.9$ are $0.2057,0.3368,0.4496,0.6458$, and 0.9895 , corresponding to $x=6, \ldots, 10$. In total, there are 10 points for the solutions. By Procedure 1, we need to calculate the coverage probabilities at these 10 values of $\theta$. The probabilities are $0.1,0.9129,0.9494,0.9627,0.8926,0.8926,0.9627$, $0.9494,0.9129$, and 0.1 , corresponding to these 10 points. The minimum value of these 10 probabilities is 0.1 ; therefore, the minimum coverage probability of this TI is 0.1 .

Example 5.2 (Example 5.1 continued). When applying Procedure 2 , it is necessary to calculate the functions for $h_{i}(\theta)$, which are $h_{0}(\theta)=\sum_{i=0}^{5} l_{\theta}(i), h_{1}(\theta)=\sum_{i=1}^{5} l_{\theta}(i), h_{2}(\theta)=$ $\sum_{i=1}^{6} l_{\theta}(i), h_{3}(\theta)=\sum_{i=1}^{7} l_{\theta}(i), h_{4}(\theta)=\sum_{i=2}^{7} l_{\theta}(i), h_{5}(\theta)=$ $\sum_{i=2}^{8} l_{\theta}(i), h_{6}(\theta)=\sum_{i=3}^{8} l_{\theta}(i), h_{7}(\theta)=\sum_{i=3}^{9} l_{\theta}(i), h_{8}(\theta)=$ $\sum_{i=4}^{9} l_{\theta}(i), h_{9}(\theta)=\sum_{i=5}^{9} l_{\theta}(i)$, and $h_{10}(\theta)=\sum_{i=5}^{10} l_{\theta}(i)$, corresponding to $v_{0}=0, v_{1}=0.0105, \ldots, v_{9}=0.7943$, and $v_{10}=$ 0.9895 . Then, by numerical calculation,

$$
\begin{aligned}
S=\int_{0}^{0.0105} h_{0}(\theta) d \theta+\int_{0.0105}^{0.2057} h_{1}(\theta) d \theta & \\
& +\cdots+\int_{0.9895}^{1} h_{10}(\theta) d \theta=0.8228
\end{aligned}
$$

is the exact average coverage probability. 
Table 1. Minimum and average coverage probabilities for the $(0.9,0.95)$ two-sided TIs for the binomial distribution based on (3) and (4) with different sample sizes, $n$

\begin{tabular}{|c|c|c|c|c|}
\hline$n$ & $\begin{array}{c}\text { Minimum confidence } \\
\text { level of TI } \\
\text { based on (3) }\end{array}$ & $\begin{array}{c}\text { Minimum confidence } \\
\text { level of TI } \\
\text { based on (4) }\end{array}$ & $\begin{array}{c}\text { Average } \\
\text { level of TI } \\
\text { based on (3) }\end{array}$ & $\begin{array}{c}\text { Average } \\
\text { level of TI } \\
\text { based on (4) }\end{array}$ \\
\hline 5 & 0.1 & 0.9932 & 0.7063 & 0.9992 \\
\hline 10 & 0.1 & 0.9926 & 0.8228 & 0.9986 \\
\hline 15 & 0.1 & 0.9902 & 0.8774 & 0.9968 \\
\hline 20 & 0.1 & 0.9868 & 0.9001 & 0.9950 \\
\hline 25 & 0.1 & 0.9851 & 0.9130 & 0.9946 \\
\hline 30 & 0.1 & 0.9811 & 0.9242 & 0.9943 \\
\hline 35 & 0.1 & 0.9855 & 0.9293 & 0.9946 \\
\hline 40 & 0.1 & 0.9846 & 0.9363 & 0.9938 \\
\hline 45 & 0.1 & 0.9835 & 0.9407 & 0.9932 \\
\hline 50 & 0.1 & 0.9839 & 0.9439 & 0.9930 \\
\hline
\end{tabular}

The Matlab programs for computing the minimum and average coverage probabilities for binomial and Poisson distributions are available from the authors on request.

Tables 1 and 2 list the minimum and average coverage probabilities corresponding to different sample sizes, $n$, based on the confidence intervals in (3) and (4).

Note that the performance of the lower tolerance bound is the same as that of the upper tolerance bound. Tables 1 and 2 show that for the TI based on (3), the two-sided TI and the one-sided tolerance bound have very similar performance. The minimum coverage probability is 0.1 for any sample size, far away from the nominal level of 0.95 . This indicates that the minimum coverage probability cannot be improved by increasing the sample size, and that the coverage probability is near 0.1 when the true proportion, $\theta$, is near the point at which the minimum coverage probability occurs. Although the minimum coverage probability is much lower than the nominal level, its average coverage probability increases as the sample size increases, and it can reach the nominal level when the sample size is not small. If we use the average coverage probability as a criterion to evaluate TIs, then the TI based on (3) performs well when $n$ is not small; but if we use the minimum coverage probability as a criterion, then this TI can be criticized for its lower minimum coverage probability.
Remark 4. The minimum coverage probability based on (3) is 0.1 regardless of sample size, because the point in the parameter space at which the minimum coverage probability occurs is a variable function of the sample size. This point decreases to 0 or increases to 1 as the sample size increases. In this case, regardless of the sample size, there exists a point in the parameter space with coverage probability 0.1 .

Tables 1 and 2 show that for the TI based on (4), unlike the TI based on (3), the performance of the two-sided interval is not very similar to that of the upper bound. The minimum coverage probability and the average coverage probability of the TI based on (4) are equal to or larger than the nominal level, 0.95 , for all $n$. Because its minimum coverage probability is close to its average coverage probability, the performance of this TI is rather stable for each proportion, $\theta$, in the parameter space. This large difference between the minimum coverage probabilities for the two TIs is similar to that seen in confidence interval estimation, because in the confidence interval estimation, the coverage probability of (4) can reach $1-\alpha$, even though the infimum coverage probability of the Wald interval (3) is 0.

We may propose to construct an improved TI based on this TI such that the minimum or average coverage probability is close to the nominal level by choosing a smaller $\alpha$ value in the first step of the two-step procedure. Details on how to choose a smaller $\alpha$ value are given in the next section.

Table 2. The minimum and average coverage probabilities for the $(0.9,0.95)$ upper tolerance bound for a binomial distribution based on (3) and (4) with different sample sizes, $n$

\begin{tabular}{|c|c|c|c|c|}
\hline$n$ & $\begin{array}{c}\text { Minimum confidence } \\
\text { level of TI } \\
\text { based on (3) }\end{array}$ & $\begin{array}{c}\text { Minimum confidence } \\
\text { level of TI } \\
\text { based on (4) }\end{array}$ & $\begin{array}{c}\text { Average } \\
\text { level of TI } \\
\text { based on (3) }\end{array}$ & $\begin{array}{c}\text { Average } \\
\text { level of TI } \\
\text { based on (4) }\end{array}$ \\
\hline 5 & 0.1 & 0.9932 & 0.8484 & 0.9996 \\
\hline 10 & 0.1 & 0.9554 & 0.8876 & 0.9921 \\
\hline 15 & 0.1 & 0.9523 & 0.9140 & 0.9897 \\
\hline 20 & 0.1 & 0.9591 & 0.9265 & 0.9892 \\
\hline 25 & 0.1 & 0.9519 & 0.9326 & 0.9867 \\
\hline 30 & 0.1 & 0.9505 & 0.9400 & 0.9817 \\
\hline 35 & 0.1 & 0.9529 & 0.9400 & 0.9822 \\
\hline 40 & 0.1 & 0.9504 & 0.9422 & 0.9812 \\
\hline 45 & 0.1 & 0.9504 & 0.9437 & 0.9788 \\
\hline 50 & 0.1 & 0.9504 & 0.9441 & 0.9791 \\
\hline
\end{tabular}




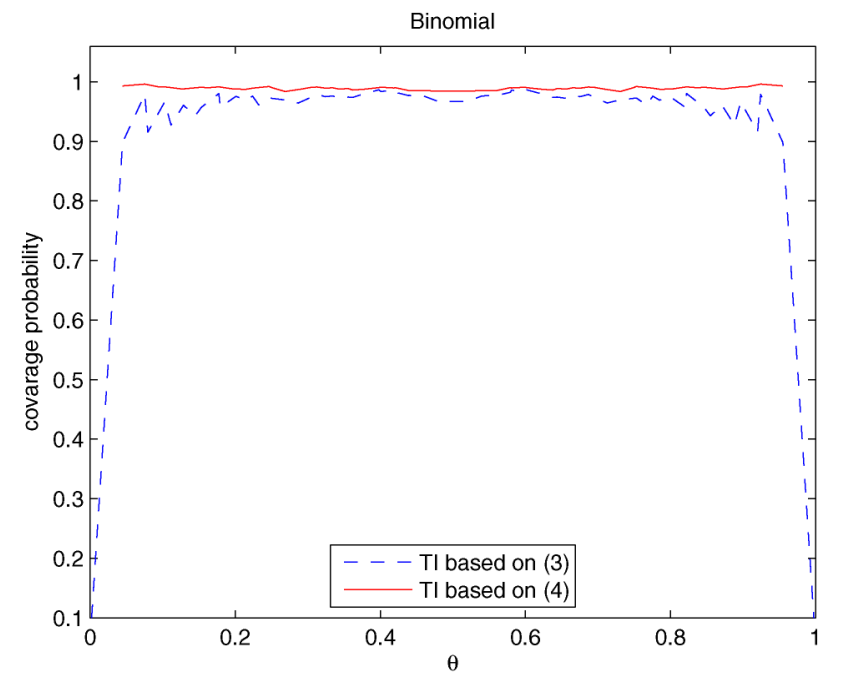

Figure 1. Coverage probabilities of the tolerance intervals based on (3) and (4).

Figures 1 and 2 give the plots of the coverage probabilities of the two-sided $(0.9,0.95)$ TIs for a binomial distribution with $n=50$ and a Poisson distribution with $\theta \leq 30$.

These plots show that for the binomial distribution, the coverage probability of the TI based on (3) is lower than that based on (4), and for the Poisson distribution, the coverage probability based on (5) also is lower than that based on (6). But in both distributions, the minimum and average coverage probabilities of the TIs are either lower or higher than the nominal level, except when the lower and upper tolerance bounds are based on (4) (see Tables 1 and 2 and Figures 1 and 2).

In the next section we propose improved TIs to meet the required coverage probability based on the procedures outlined in Sections 3 and 4 .

\section{IMPROVED TOLERANCE INTERVALS}

From the numerical results given in Section 5, we know that the coverage probabilities for most of the existing TIs of the

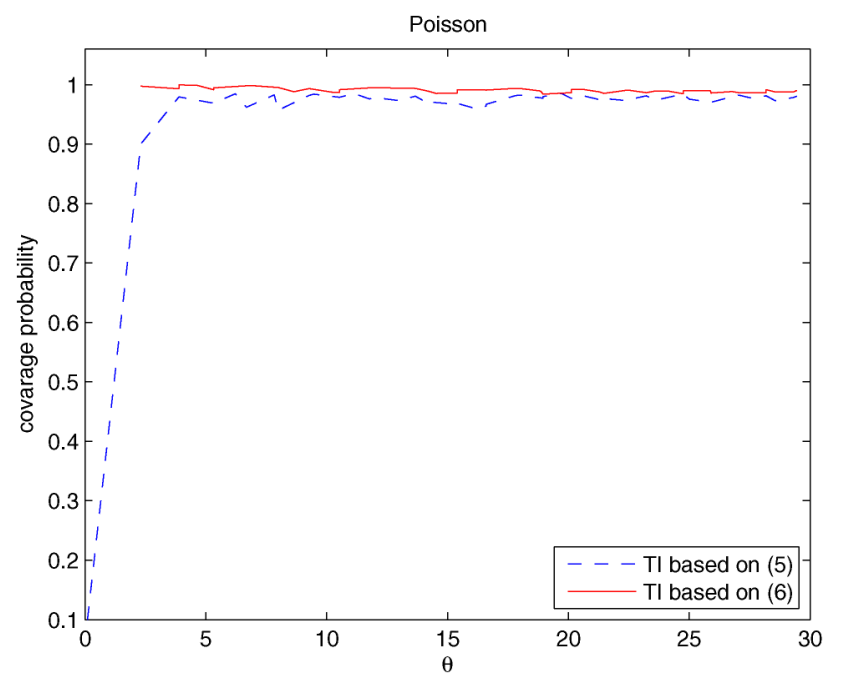

Figure 2. Coverage probabilities of the tolerance intervals based on (5) and (6). binomial and Poisson distributions in the literature are either much higher or much lower than the nominal level. In this section we construct improved TIs based on Procedure 1 or Procedure 2 such that the minimum or average coverage probabilities can be close to the nominal level. For the binomial distribution, the approach is to choose a smaller $\alpha$ value in the confidence interval (4), such that the minimum coverage probability or the average coverage probability of the TIs constructed from the two-step procedure are equal to the nominal level. The Poisson distribution can be determined similarly. If we adopt the minimum coverage probability criterion, then we can use Procedure 1 to calculate the minimum coverage probability for different $\alpha$ and choose an $\alpha$ such that its minimum coverage probability is close to the nominal level. If we adopt the average coverage probability criterion, then we can use Procedure 2 to calculate the average coverage probability for different $\alpha$ and choose an $\alpha$ such that its average coverage probability is close to the nominal level. The appropriate $\alpha$ s for different sample sizes are presented in Tables 3-5. Note that from Table 2, the minimum coverage probabilities of the upper tolerance bounds based on (4) are close to the nominal level; thus in this case, we do not need to search for other $\alpha$ values, and we simply use the conventional $\alpha$ value of 0.05 .

Because both minimum and average coverage probability criteria are given and two sets of $\alpha$ are suggested, we may be interested in which criterion we should use. This choice may depend on the tolerance in each case. For example, as described by Hahn and Meeker (1991), in response to a request by a regulatory agency, a manufacturer must provide a statement concerning whether the maximum noise limit, under specified operating conditions, is met by a high proportion of units, such as $95 \%$ of a particular model of a jet engine. In this case, the manufacturer desires a one-sided upper $90 \%$ tolerance bound that will be met by at least $95 \%$ of its jet engines. In this example, if we use the minimum coverage probability criterion, then we require that for an upper $90 \%$ tolerance bound, $U_{m}$, the noise limit of at least $95 \%$ of the units of each product be less than the upper bound, $U_{m}$. If we use the average coverage probability criterion, then we require that for an upper $90 \%$ tolerance bound, $U_{a}$, the noise limit of an average of $95 \%$ of the units of all products be less than the upper bound, $U_{a}$. It is obvious that $U_{a}<U_{m}$. If the agency cannot accept the upper tolerance bound $U_{m}$ but can accept $U_{a}$, then we may use the second criterion to report to the agency that the products meet the noise requirement under the second criterion. However, if the agency requires that at least 95\% of units of each product must meet the requirement, then we must use the minimum coverage probability criterion and produce the products that satisfy the requirement.

\section{ILLUSTRATIVE EXAMPLES}

The binomial and Poisson TIs are useful in many industrial applications. Here we use two real examples for illustration.

A Binomial Example. The first example is from a semiconductor manufacturing process. The locations of chips on a wafer as measured on 30 wafers. On each wafer, 50 chips are measured, and a defective wafer is identified whenever a misregistration, in terms of horizontal and/or vertical distance from 
Table 3. Suggested $\alpha$ values in (4) such that the minimum coverage probability for the $(0.9,0.95)$ two-sided TI for the binomial distribution based on (4) is close to the nominal level, 0.95, as well as their corresponding minimum and average coverage probabilities for different sample sizes, $n$

\begin{tabular}{lccc}
\hline \hline$n$ & $\alpha$ & $\begin{array}{c}\text { Minimum coverage } \\
\text { probability }\end{array}$ & $\begin{array}{c}\text { Average coverage } \\
\text { probability }\end{array}$ \\
\hline 10 & 0.25 & 0.9494 & 0.9842 \\
15 & 0.17 & 0.9593 & 0.9847 \\
20 & 0.16 & 0.9449 & 0.9800 \\
25 & 0.16 & 0.9546 & 0.9793 \\
30 & 0.15 & 0.9498 & 0.9779 \\
35 & 0.13 & 0.9514 & 0.9789 \\
40 & 0.12 & 0.9582 & 0.9815 \\
45 & 0.12 & 0.9574 & 0.9793 \\
50 & 0.12 & 0.9562 & 0.9784 \\
\hline
\end{tabular}

the center, is recorded. The defective number follows a binomial distribution, $\mathrm{B}(50, \theta)$. The data can be obtained from the NIST/SEMATECH e-Handbook of Statistical Methods: $h t t p: / /$ www.itl.nist.gov/div898/handbook/pmc/section3/pmc332.htm.

Note that because the original data are overdispersed, we use part of the data in this example. These data are given in Table 6.

The tolerance intervals for the data set based on (3) and (4) are $(1,20)$ and $(1,21)$.

From these data, because the fraction of defective wafers ranges between 0.1 and 0.26 , we may assume that the parameter space is restricted to $(0,0.4)$ instead of $(0,1)$. The minimum coverage probability of the two $(0.9,0.95)$ two-sided TIs based on (3) and (4) for $\theta \in(0,0.4)$ can be derived by Procedure 1, which is the minimum value of the coverage probabilities for the solutions in Step 2 belonging to $(0,0.4)$ by Remark 2. By calculation, the minimum coverage probabilities for TIs based on (3) and (4) are 0.1 and 0.9839 , which occur at $\theta$ near 0.02 and at $\theta$ near 0.048 . The average coverage probabilities for TIs based on (3) and (4) are 0.9345 and 0.9937. Because the true fraction of defective wafers could be near 0.02 , the coverage probability of the TI based on (3) is only 0.1 , which is much lower than the nominal level of 0.95 . In this case the TI based on (4) is more acceptable than that based on (3) from the standpoint of the minimum coverage probability. Note that

Table 4. Suggested $\alpha$ values in (4) such that the average coverage probability for the $(0.9,0.95)$ two-sided TI for a binomial distribution based on (4) is close to the nominal level, 0.95, as well as their corresponding minimum and average coverage probabilities for different sample sizes, $n$

\begin{tabular}{lccc}
\hline \hline$n$ & $\alpha$ & $\begin{array}{c}\text { Minimum coverage } \\
\text { probability }\end{array}$ & $\begin{array}{c}\text { Average coverage } \\
\text { probability }\end{array}$ \\
\hline 10 & 0.37 & 0.7985 & 0.9506 \\
15 & 0.34 & 0.8705 & 0.9488 \\
20 & 0.29 & 0.8874 & 0.9509 \\
25 & 0.27 & 0.8911 & 0.9495 \\
30 & 0.27 & 0.8911 & 0.9501 \\
35 & 0.25 & 0.8923 & 0.9497 \\
40 & 0.23 & 0.8873 & 0.9506 \\
45 & 0.24 & 0.8910 & 0.9505 \\
50 & 0.22 & 0.9160 & 0.9523 \\
\hline
\end{tabular}

Table 5. Suggested $\alpha$ values in (4) such that the average coverage probability for the $(0.9,0.95)$ upper tolerance bound for a binomial distribution based on (4) is close to the nominal level, 0.95, as well as their corresponding minimum and average coverage probabilities for different sample sizes, $n$

\begin{tabular}{lccc}
\hline \hline$n$ & $\alpha$ & $\begin{array}{c}\text { Minimum coverage } \\
\text { probability }\end{array}$ & $\begin{array}{c}\text { Average coverage } \\
\text { probability }\end{array}$ \\
\hline 10 & 0.22 & 0.7928 & 0.9543 \\
15 & 0.16 & 0.8457 & 0.9556 \\
20 & 0.15 & 0.8562 & 0.9505 \\
25 & 0.13 & 0.8705 & 0.9496 \\
30 & 0.12 & 0.8828 & 0.9516 \\
35 & 0.12 & 0.8853 & 0.9525 \\
40 & 0.10 & 0.9009 & 0.9543 \\
45 & 0.10 & 0.9014 & 0.9538 \\
50 & 0.10 & 0.9007 & 0.9516 \\
\hline
\end{tabular}

if we choose a wider parameter space that includes the interval $(0,0.4)$, then the minimum coverage probability of the TI based on (3) is still 0.1 , and the minimum coverage probability of the TI based on (4) is close to 0.9839 , as given in Table 1 . We still conclude that the TI based on (4) is more acceptable than that based on (3).

If the true parameter is known to belong to a subset, like $(0.154,0.400)$, then, by calculation, the minimum coverage probabilities for TIs based on (3) and (4) are 0.9573 and 0.991. The average coverage probabilities for TIs based on (3) and (4) are 0.9774 and 0.9917 . In this case the TI based on (3) is acceptable. This indicates that the performance of the TI also depends on the parameter space.

A Poisson Example. The second example is from a steel manufacturing process (Montgomery 1996). We consider part of the data set for this example. Here the surface defects are counted on 21 rectangular steel plates. The 21 defect counts, following the Poisson distribution, $P(\lambda)$, are

$$
1,0,4,3,1,2,0,2,1,1,0,0,2,1,3,4,3,1,0,2,4 .
$$

The TIs based on (5) and (6) for the data are $(0,9)$ and $(0,12)$.

The numbers in the data range between 0 and 4 . We may consider a parameter space for the true mean of the Poisson distribution with a wider range of $(0,9)$. By Procedure 1 and Remark 2, the minimum coverage probabilities of the two $(0.9,0.95)$ two-sided TIs based on (5) and (6) for $\lambda \in(0,9)$ can be derived. The minimum coverage probabilities are 0.1 and 0.9870 for intervals based on (5) and (6). Their corresponding average coverage probabilities are 0.8806 and 0.9966 . This

Table 6. The semiconductor data

\begin{tabular}{lccccc}
\hline \hline $\begin{array}{l}\text { Sample } \\
\text { number }\end{array}$ & $\begin{array}{c}\text { Fraction } \\
\text { defective }\end{array}$ & $\begin{array}{c}\text { Sample } \\
\text { number }\end{array}$ & $\begin{array}{c}\text { Fraction } \\
\text { defective }\end{array}$ & $\begin{array}{c}\text { Sample } \\
\text { number }\end{array}$ & $\begin{array}{c}\text { Fraction } \\
\text { defective }\end{array}$ \\
\hline 1 & 0.24 & 11 & 0.10 & 21 & 0.22 \\
2 & 0.16 & 12 & 0.12 & 22 & 0.18 \\
3 & 0.20 & 13 & 0.24 & 23 & 0.24 \\
4 & 0.14 & 14 & 0.16 & 24 & 0.14 \\
5 & 0.18 & 15 & 0.20 & 25 & 0.26 \\
6 & 0.28 & 16 & 0.10 & 26 & 0.18 \\
7 & 0.20 & 17 & 0.26 & 27 & 0.12 \\
\hline
\end{tabular}


means that the coverage probability of the first TI is too far away from its nominal coverage probability, and that the second $\mathrm{TI}$ is too conservative, because its minimum coverage probability is much larger than its nominal level of 0.95 . As in the binomial distribution, the performance of the TIs depends on the parameter space.

In both examples, the conventional TIs perform unsatisfactorily. We suggest using the improved TIs proposed in Section 6 for these two examples.

For the binomial distribution, the $\alpha$ value for the improved $\mathrm{TI}$ in Table 3 for sample size $n=50$ is 0.12 when the parameter space is $(0,1)$. In this binomial example, because the possible parameter space is $(0,0.4)$, by Remark 2 , we can find an $\alpha$ value such that the minimum coverage probability of the TI based on (4) in this restricted parameter is close to 0.95 . By computation, the suggested $\alpha$ value is 0.12 , the same as the $\alpha$ value for the natural parameter space $(0,1)$. The improved TI for the binomial data set with respect to $\alpha=0.12$ is $(1,17)$. The minimum and average coverage probabilities with respect to this parameter space are 0.9562 and 0.9791 .

For this Poisson example, by computation using Remark 2, the suggested $\alpha$ value for the TI based on (6) is 0.17 when the restricted parameter space is $(0,9)$. The improved TI is $(0,10)$. The minimum and average coverage probabilities with respect to this parameter space are 0.9493 and 0.9792 .

Remark 5. In a real application, if the data are overdispersed, then estimated improved tolerance limits can be found by separating the data into several groups such that the data are not overdispersed in each group, then calculating the tolerance limits for the data in each group. The largest upper tolerance and smallest lower tolerance limits provide the estimated upper and lower tolerance limits for the full data set.

\section{CONCLUSION}

The minimum and average coverage probabilities are two important indexes for evaluating the performance of TIs. Obtaining the exact values for these two probabilities is critical. Here we have proposed the first method for computing the exact minimum and average coverage probabilities for TIs of binomial and Poisson distributions.

With the procedures proposed in this article, we need only calculate the coverage probabilities at some finite points in the parameter space. Then the exact values can be derived by making inferences on these finite coverage probabilities. As demonstrated by our simulation studies, our procedures definitely provide a more accurate and efficient way to achieve this goal.

Procedures 1 and 2 allow precise calculation of the minimum and average coverage probabilities of the conventional TIs in the literature. Our results indicate that most of the TIs are higher or lower than the nominal level. Because inaccurate coverage probability information will lead to serious losses, regardless of whether it is an overestimation or an underestimation, our proposed procedures may be of great help in precisely reporting the TI information and helping both the manufacturer and customer make informed decisions.

We also have specified improved TIs based on the proposed procedures. These TIs can obtain the required coverage probability regardless of sample size, because the $\alpha$ value is modified depending on the sample size when constructing TIs. With these improved TIs, we need not worry about whether the true coverage probability is higher or lower than the nominal level, because we can let either their minimum or average coverage probability be very close to the nominal level. Both the manufacturer and customer will surely benefit from the use of these improved TIs.

\section{ACKNOWLEDGMENTS}

This research was supported by RGC Competitive Earmarked Research grants 620606 and 620707. The authors thank the editor, an associate editor, and three anonymous referees for their insightful and helpful comments.

\section{APPENDIX: PROOFS}

\section{Proof of Lemma 1}

We need to show that $\sum_{i=L(k)}^{U(k)} f_{\theta}(i)$ and $\sum_{i=L(l)}^{U(l)} f_{\theta}(i)$ have one solution. Note that

$$
\sum_{i=L(k)}^{U(k)} f_{\theta}(i)-\sum_{i=L(l)}^{U(l)} f_{\theta}(i)=\sum_{i=L(k)}^{L(l)-1} f_{\theta}(i)-\sum_{i=U(k)+1}^{U(l)} f_{\theta}(i) .
$$

By the order of $\theta$ in (11), the first term in (11) is larger than the second term in (11) when $\theta$ goes to 0 , and the reverse is true when $\theta$ goes to 1 . Note that the first term in (11) is a decreasing function or a unimodal function, and that the second term in (11) is an increasing function or a unimodal function. Consequently, there is only one solution for (11) set to 0 .

\section{Proof of Lemma 2}

(a) If $L(x)=L(y)=0$, then, by assumption, we have $L(z)=0$ if $x<z<y$. Because $\sum_{i=0}^{U(x)} f_{\theta}(i), \sum_{i=0}^{U(y)} f_{\theta}(i)$, and $\sum_{i=0}^{U(z)} f_{\theta}(i)$ are decreasing functions by Proposition 1 and $\sum_{i=0}^{U(x)} f_{\theta}(i) \leq \sum_{i=0}^{U(z)} f_{\theta}(i) \leq \sum_{i=0}^{U(y)} f_{\theta}(i)$, we have $q_{x}<q_{z}<q_{y}$.

(b) If $U(x)=U(y)=n$, then, by assumption, we have $U(z)=n$. Because $\sum_{i=L(x)}^{n} f_{\theta}(i), \sum_{i=L(y)}^{n} f_{\theta}(i)$ and $\sum_{i=L(z)}^{n} f_{\theta}(i)$ are increasing functions, and $\sum_{i=L(X)}^{n} f_{\theta}(i) \geq \sum_{i=L(Z)}^{n} f_{\theta}(i) \geq$ $\sum_{i=L(Y)}^{n} f_{\theta}(i)$, we have $q_{x}<q_{z}<q_{y}$.

(c) For cases other than (a) and (b), if we can show that the equation $\sum_{i=L(k)}^{U(k)} f_{\theta}(i)=\sum_{i=L(l)}^{U(l)} f_{\theta}(i)$ has only one solution, and the value for $\theta_{x}$ is an increasing function of $x$, where $\theta_{x}$ occurs at the maximum value of $\sum_{i=L(x)}^{U(x)} f_{\theta}(i)$, then $p_{x}$ and $q_{x}$ are increasing functions of $x$. If $p_{y}<p_{x}$ and $\theta_{x}<\theta_{y}$, it follows that the equation $\sum_{i=L(x)}^{L(x)} f_{\theta}(i)=\sum_{i=L(y)}^{U(y)} f_{\theta}(i)$ has two solutions. By Lemma $1, \sum_{i=L(x)}^{L(x)} f_{\theta}(i)=\sum_{i=L(y)}^{U(y)} f_{\theta}(i)$ has only one solution; therefore, $p_{x}$ and $q_{x}$ are increasing functions of $x$. Consequently, we have $p_{x}<p_{z}<p_{y}$ and $q_{x}<q_{z}<q_{y}$.

\section{Proof of Theorem 1}

Assume that there are totally $k$ points of $p_{i}$ and $q_{i}$. We rank the $k$ endpoints from the smallest to the largest. Let $v_{i}$, $i=1, \ldots, k$, denote the $i$ th smallest value of these endpoints. For a $\theta \in\left(v_{i}, v_{i+1}\right)$, let $k_{0}(\theta)$ denote the smallest $x$ such that $\theta<q_{x}$ and $k_{1}(\theta)$ denote the largest $x$ such that $p_{x}<\theta$. By 
Lemma 2, the coverage probability of the interval at a parameter, $\theta$, is

$$
P_{\theta}(F(U(X))-F(L(X))>\beta)=\sum_{i=k_{0}(\theta)}^{k_{1}(\theta)} f_{\theta}(i) .
$$

We show that

$$
\begin{aligned}
\inf _{v_{i}<\theta<v_{i+1}} P_{\theta}(F(U(X))-F(L(X))>\beta) & \\
=\min _{\theta=v_{i} \text { orv }} P_{i+1} & P_{\theta}(F(U(X))-F(L(X))>\beta) .
\end{aligned}
$$

With this result, the infimum of the coverage probabilities for $\theta \in\left(v_{i}, v_{i+1}\right)$ is the minimum of the coverage probabilities $P_{v_{i}}(F(U(X))-F(L(X))>\beta)$ and $P_{v_{i+1}}(F(U(X))-F(L(X))>$ $\beta)$. Thus, when (13) holds, the infimum of the coverage probabilities for $\theta$ in the parameter space is the minimum of the coverage probabilities, $\left\{P_{\theta}(F(U(X))-F(L(X))>\beta): \theta \in W\right\}$.

Now we prove (13). Note that for a fixed $i, k_{0}(\theta)$ and $k_{1}(\theta)$ are constants for all $\theta \in\left(v_{i}, v_{i+1}\right)$, because there are no other $p_{x}$ and $q_{x}$ between $v_{i}$ and $v_{i+1}$. According to Proposition 1, (12) is a unimodal function, a decreasing function or an increasing function of $\theta$. Thus, by the properties of these functions, the minimum value of $\inf _{v_{i}<\theta<v_{i+1}} P_{\theta}(F(U(X))-F(L(X))>\beta)$ occurs at $\theta=v_{i}$ or $v_{i+1}$. Thus the proof is complete.

\section{Proof of Theorem 2}

By a similar argument as in the proof of Theorem 1, the coverage probability of (12) for $\theta$ belonging to an interval $\left(v_{i}, v_{i+1}\right)$ is $\sum_{j=a_{i}}^{b_{i}} f_{\theta}(j)$ for some $a_{i}$ and $b_{i}$ depending on $i$. By definition, when $v_{i}$ is a $p_{x}$, the maximum value and the minimum value of $y$ such that $F(U(y))-F(L(y)) \geq \beta$ for $\theta \in\left(v_{i}, v_{i+1}\right)$ are $x$ and $r_{i}$. When $v_{i}$ is a $q_{x}$, the maximum value and the minimum value of $y$ such that $F(U(y))-F(L(y)) \geq \beta$ for $\theta \in\left(v_{i}, v_{i+1}\right)$ are $s_{i}$ and $x+1$. By Lemma 2, when $v_{i}$ is a $p_{x}$, we have $F(U(y))-F(L(y)) \geq \beta$ for $\theta \in\left(v_{i}, v_{i+1}\right)$ and $r_{i} \leq y \leq x$, and when $v_{i}$ is a $q_{x}$, we have $F(U(y))-F(L(y)) \geq \beta$ for $\theta \in\left(v_{i}, v_{i+1}\right)$ and $x+1 \leq y \leq s_{i}$. Therefore, the proof is completed.

[Received March 2007. Revised June 2008.]

\section{REFERENCES}

Agresti, A., and Coull, B. (1998), “Approximate Is Better Than 'Exact' for Interval Estimation of Binomial Proportions," The American Statistician, 52, 119-126.

Brown, L. D., Cai, T. T., and DasGupta, A. (2001), "Interval Estimation for a Binomial Proportion" (with discussion), Statistical Science, 16 (2), 101133

Fernholz, L. T., and Gillespie, J. A. (2001), "Content-Correct Tolerance Limits Based on the Bootstrap," Technometrics, 43 (2), 147-155.

Hahn, G. J., and Chandra, R. (1981), "Tolerance Intervals for Poisson and Binomial Variables,' Journal of Quality Technology, 13 (2), 100-110.

Hahn, G. J., and Meeker, W. Q. (1991), Statistical Intervals: A Guide for Practitioners, New York: Wiley.

Hamada, M., Johnson, V., Moore, L. M., and Wendelberger, J. (2004), "Bayesian Prediction Intervals and Their Relationship to Tolerance Intervals," Technometrics, 46 (4), 452-459.

Krishnamoorthy, K., and Mathew, T. (2004), "One-Sided Tolerance Limits in Balanced and Unbalanced One-Way Random Models Based on Generalized Confidence Intervals," Technometrics, 46 (1), 44-52.

Liao, C. T., Lin, T. Y., and Iyer, H. K. (2005), "One- and Two-Sided Tolerance Intervals for General Balanced Mixed Models and Unbalanced One-Way Random Effects Model," Technometrics, 47 (3), 323-335.

Montgomery, D. C. (1996), Introduction to Statistical Quality Control (3rd ed.), New York: Wiley.

Odeh, R. E., and Owen, D. B. (1980), Tables for Normal Tolerance Limits, Sampling Plans, and Screening, New York: Dekker.

Van Der Merwe, A. J., Pretorius, A. L., and Meyer, J. H. (2006), "Bayesian Tolerance Intervals for the Unbalanced One-Way Random Effects Model," Journal of Quality Technology, 38 (3), 280-293.

Wald, A., and Wolfowitz, J. (1946), "Tolerance Limits for Normal Distribution," Annals of Mathematical Statistics, 17, 208-215.

Wang, C. M., and Iyer, H. K. (1994), “Tolerance Intervals for the Distribution of True Values in the Presence of Measurement Error," Technometrics, 36, $162-170$.

Wang, H. (2007), "Exact Confidence Coefficients of Confidence Intervals for a Binomial Proportion," Statistica Sinica, 17, 361-368.

Wolfinger, R. D. (1998), "Tolerance Intervals for Variance Component Models Using Bayesian Simulation,” Journal of Quality Technology, 30 (1), 18-32.

Zacks, S. (1970), "Uniformly Most Accurate Upper Tolerance Limits for Monotone Likelihood Ratio Families of Discrete Distributions," Journal of American Statistical Association, 65, 307-316. 